\title{
MUSEUM, MOUNTING IN OPHTHALMOLOGY*
}

BY

\author{
NORMAN ASHTON
}

\author{
Department of Pathology, Institute of Ophthalmology, London
}

THE introduction of transparent plastic materials has stimulated a new interest in the techniques of museum mounting and there are few departments of pathology which have not already abandoned glass museum jars for those composed of Perspex. At the Institute of Ophthalmology the valuable collection of specimens which has resulted from the merging of the museums of the Moorfields, Westminster and Central Eye hospitals, is now being re-catalogued and re-mounted in transparent plastic containers. The purpose of this paper is to give an account of the techniques used, which are based upon the methods employed by other workers, in order that those interested in mounting museum specimens of the eye may have ready access to the various methods available. The majority of the specimens are being re-mounted in a fluid medium in Perspex containers, but solid media such as "Marco S.B. 26C" Resin and Perspex have been used in selected cases, and the results and techniques employed are set out in detail below.

Transparent Perspex Boxes. $†$-Perspex boxes of length $2 \frac{3}{4}$ in., width 2 in., depth 1 in., and thickness $\frac{1}{8}$ in. (Fig. 1) are carefully cleaned, and the back plate, which is sealed in position after the mounted specimen has been placed in the container, is similarly prepared.

A number-plate of opal Perspex is stencilled in indian ink, allowed to dry at room temperature, and then given a coating of Perspex cement on the stencilled side only; it is then inserted so that, when held in position by a single Perspex block internally, the museum number is clearly visible at the lower end of the front of the box (Fig. 5).

Thread holes are now drilled with a twist drill through the thinner centre plate $\left(\frac{1}{16}\right.$ in.) and the specimen is firmly tied into position with superfine Nylon sutures (Fig. 3). According to the size of the specimen small Perspex blocks are cut, trimmed, and-polished, and fixed in position with Perspex cement. The plate upon which the specimen is mounted is thus held in position by four blocks: lower and upper pairs (Fig. 2).

A hole is drilled in the right hand corner of the bottom of the box through which the container is subsequently filled.

At this stage a circular Perspex plug is prepared by using a fine flat file to reduce a piece of Perspex of convenient length and cross-sectional area of $\frac{1}{8}$ in. to a smooth rod. The plug should be sufficiently tight to permit of limited but smooth rotation within the fitting hole.

*Received for publication March 30, 1950.

†These boxes may be obtained ready-made from the "Visijar" Laboratory Supply Co., Ltd., 149, London Road, Croydon, Surrey. 


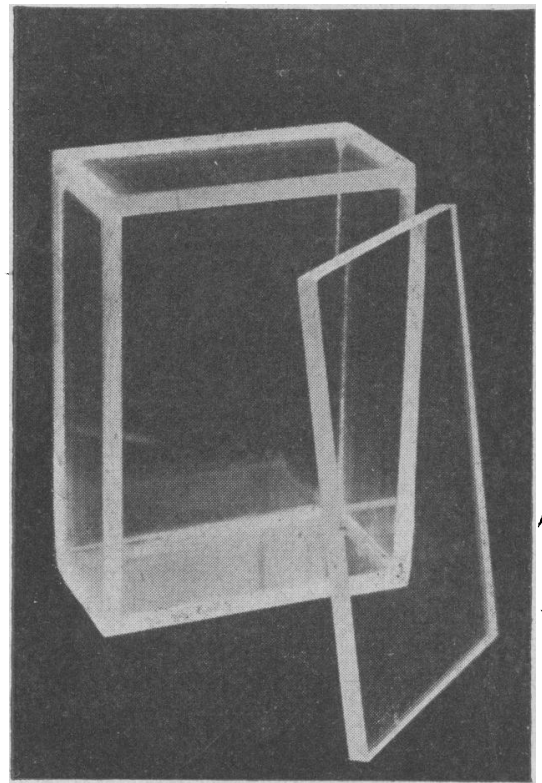

FIG. 1.-Perspex box ready for use. The back-plate is later sealed in position.

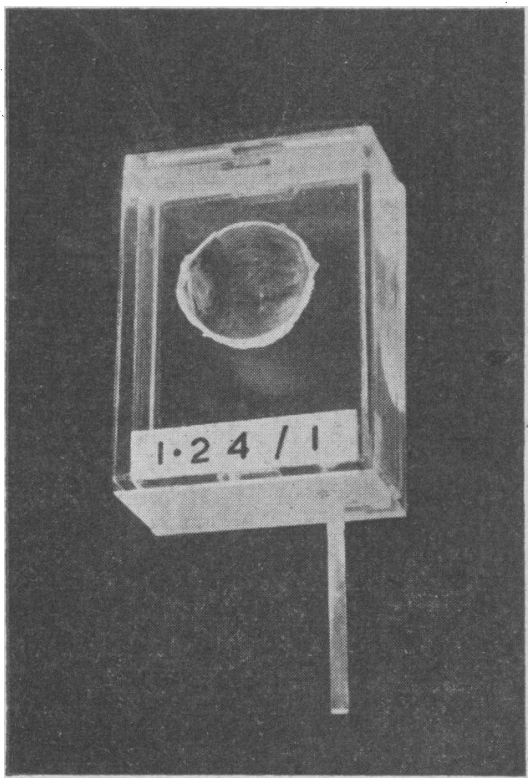

Fig. 3.-The specimen is mounted on the centre plate and tied into position with superfine Nylon sutures.

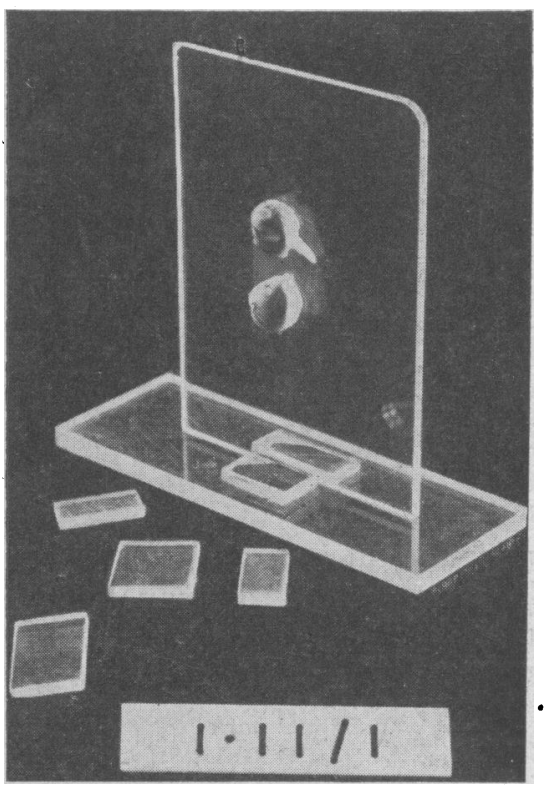

FIG. 2.-The plate upon which the specimen is mounted is held in position by four blocks: lower and upper pairs.

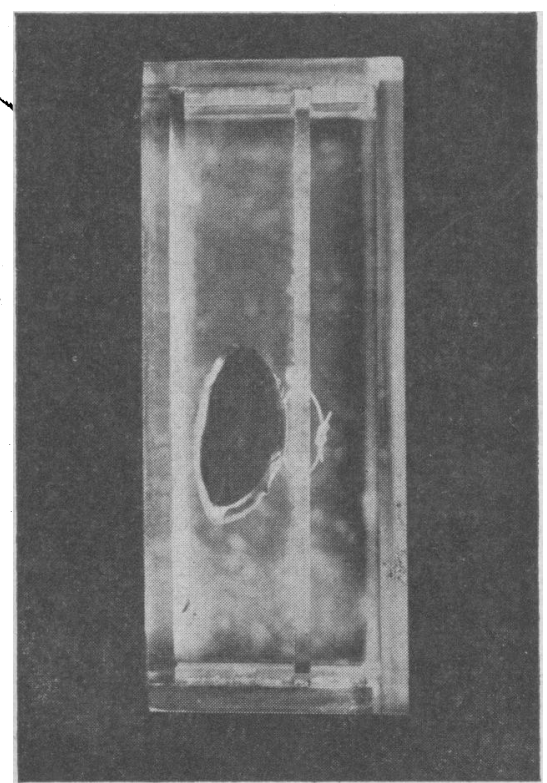

FIG. 4.-The box is filled through a hole in the bottom plate, which is later sealed with a Perspex plug. 
The back plate is fixed by clamping after softening the open edges of the box by immersion in chloroform and, when it is completely dry, the mounting fluid is slowly run in. The requisite amount of formalin is now added and finally the sodium hydrosulphite, in amount equivalent to 1 per cent. of the total solution: the crystalline solid being dissolved in a little air-free mounting fluid. The box should be gently rocked to ensure an even diffusion of the hydrosulphite. With this method the natural colours are usually restored within a few days although it may take several weeks for the colour to return completely. Where sodium hydrosulphite is used to restore the colour, Zenker's solution must not be used for fixation; otherwise the specimen will become discoloured by a black deposit.

Sealing is carried out by the following method: the last bubble is positioned below the filling hole by tilting and it is evicted by positive pressure upon the lateral and posterior surfaces of the box. After allowing the box to stand at room temperature for 24 hours, any further bubbles are removed before sealing, which is effected by means of the circular plug previously prepared. The fluid level is adjusted until it is half-way up the filling hole; a large drop of Perspex cement is placed over the hole so that the aperture is covered; the plug, moistened in chloroform and rubbed gently between the finger and thumb until an adhesive surface forms, is then plunged firmly into position (Fig. 4). The plug is left to set in situ overnight and when dry is sawn off in close proximity to the surface of the box.

The whole box now receives its final trim. All joins are uniformly rounded with the fine file; they are rendered invisible when the box is (a) coarsely polished with metal polish and (b) finely polished with liquid Perspex polish or Oakey's "Tripoli Composition" buffing soap. An electrically driven buffing wheel equipped with a loose stitched linen pad and a solid linen pad is ideal for this purpose (Fig. 5).

Mounting Fluid.-This is composed as follows: 30 per cent. glycerine, 10 per cent. sodium acetate, 0.5 per cent. formalin, water to $100 \mathrm{ml}$. It is boiled to drive off dissolved air before the formalin and sodium hydrosulphite are added. The sodium hydrosulphite in amount equivalent to 1 per cent. of the total solution is added last of all.

Solid Perspex (Methyl Methacrylate) Mounting.-The mounting of ophthalmic specimens in solid Perspex has been tried with success but the method is found to have limitations which restrict its application. The specimen is cleared so that it loses its natural appearance, and the contraction of the Perspex as it solidifies is apt to compress and distort the specimen. This method is, however, useful for mounting such specimens as portions of fragile bone, foreign bodies, and solid tumours.

Materials Required:* - These comprise methyl methacrylate monomer ("Kallodoc" I.C.I.), benzoyl peroxide (catalyst), dibutyl phthalate (plasticizer), sodium hydroxide.

During manufacture the methyl methacrylate monomer is stabilized by the addition of hydro-quinone, and this must be removed before use with caustic soda.

Washing the Monomer.-Equal quantities of monomer and 5 per cent. caustic soda are poured into a separating funnel, well fixed, and allowed to settle. The discoloured washings which form a layer below the monomer are then run off. This is repeated three times, after which the washings should be colourless. The monomer is then washed with water until the washings are neutral to phenophthalein; it is then dehydrated with flaked calcium chloride for 24 hours. After filtering through filter paper it is ready for thickening; if not required immediately it may be stored in a refrigerator.

Thickening the Monomer.-A mixture of washed monomer 85 parts (by volume), dibutyl phthalate 15 parts (by volume), and benzoyl peroxide 0.1 per cent. (by weight), is placed in a flask (which should be only half filled) and partially polymerized on a boiling water bath for 15 to 30 minutes. The time depends upon the thickness of the syrup required. Three or four shakings are necessary during the heating. These should be carried out with great care as the reaction is

* Obtainable from Imperial Chemical Industries, Gloucester House, 149, Park Lane, London, W.1. 


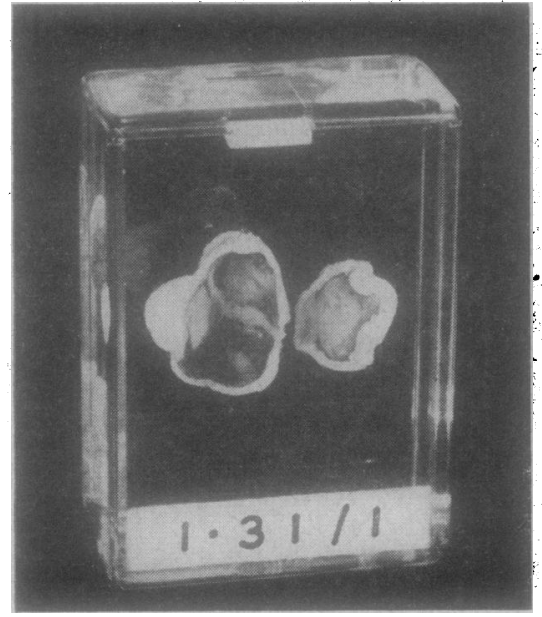

Fig. 5.-The joints of the box are rounded with a fine file and the whole container is then polished. The museum number on the inside is clearly visible.

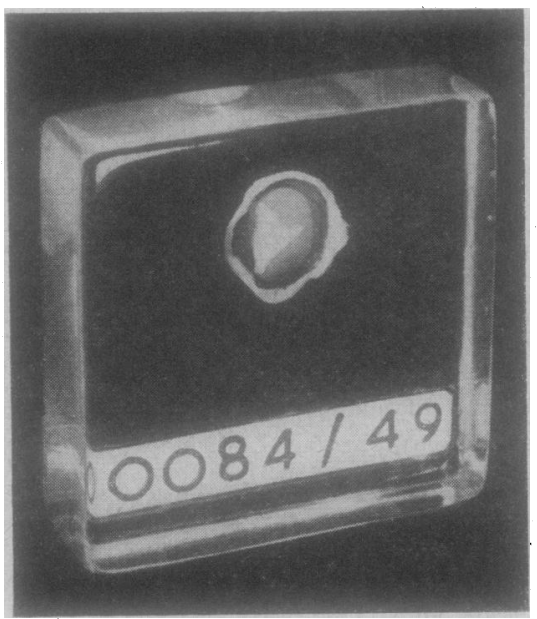

FIG. 7.--Retrolental fibroplasia. The specimen has not been cleared and is mounted in solid Marco resin.

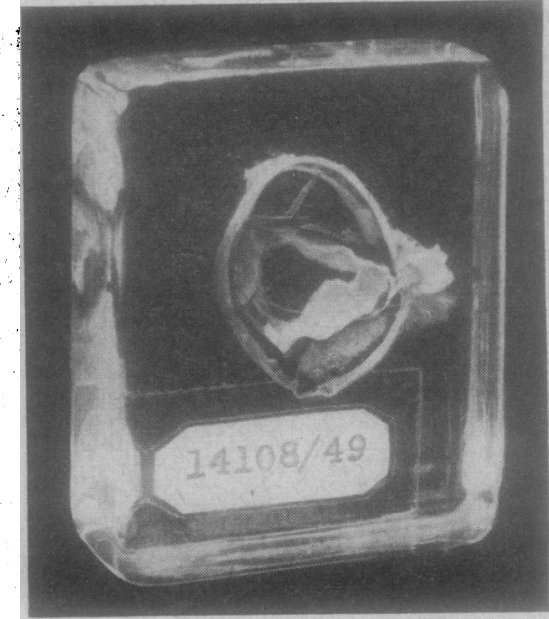

FIG. 6.-Melanoma of the choroid. The specimen is mounted in solid Perspex.

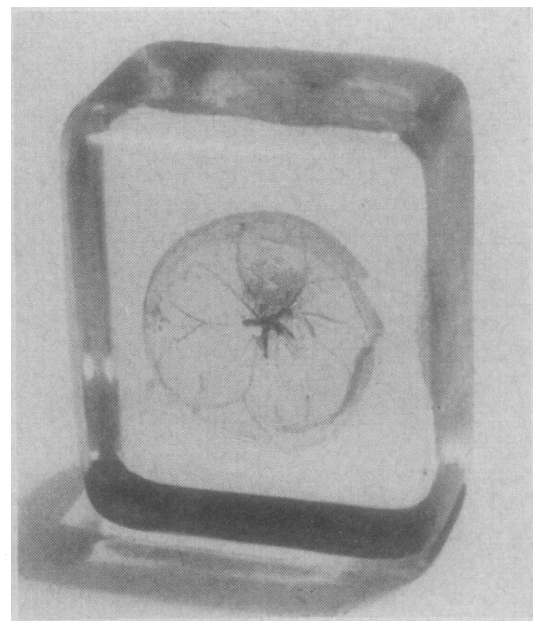

FIG. 8.-Diabetic retina injected with Indian ink; rete mirabile may be seen near the disk. The specimen is cleared and mounted whole in solid Marco resin. 
exothermic and may cause the reaction to become violent; if this should happen the flask should be plunged into a sink of cold water kept ready for the purpose. The monomer is thickened to the maximum convenient viscosity (it should be remembered that the syrup will thicken further on cooling) and stored in the refrigerator.

Moulds.-Convenient moulds are made from glass slides of appropriate size bound together with cellophane or sealed with Semmentum.

Preparation of the Specimen.-The specimen is thoroughly dehydrated (any trace of residual moisture causes clouding of the Perspex) through graded alcohols, then placed in thin monomer (1 part of thickened monomer, 3 parts of chloroform) in a jar in a vacuum tank which is evacuated by a pump giving a vacuum of 24 in. $\mathrm{Hg}$; bubbles will rise from the specimen. It should be left under vacuum for 24 hours, re-evacuating from time to time. The thin monomer is now replaced by semi-thick monomer ( 3 parts of thick monomer, 1 part of chloroform approximately) and treated by vacuum for a further 24 hours.

While the specimen is being prepared as above, a thin layer of thick monomer is poured into the mould and polymerized by ultra-violet light or heat, starting at $40^{\circ} \mathrm{C}$. and rising to $50^{\circ} \mathrm{C}$. to accelerate the final hardening. When this initial layer and the specimen are ready, the latter is transferred on to the layer of hard monomer in the mould and covered with thick monomer. The specimen is then positioned, care being taken that no bubbles are trapped underneath. The mould is then replaced in the vacuum tank, evacuated, and left under vacuum at room temperature for 24 hours : it is then polymerized under vacuum at $40^{\circ} \mathrm{C}$. until hard.

Finishing.-When hard the block is cut with a fine saw to obtain a symmetrical mount of the required size. The surface is polished with the finest possible emery papers and finished with a fine abrasive and high speed mop (Fig. 6).

Solid Resin Mounting.-Despite the yellow. colour of resin it has proved to be the best and most practical solid mounting we have tried. It is very easy to work with and quickly prepared, and specimens may be mounted cleared or uncleared as required.*

Components.-These comprise "Marco Resin S.B.26C", monomer C, diethyl phthalate (plasticizer), H.C.H. (catalyst), accelerator E.

Preparation of Plastic.-Take (A) " Marco" resin 100 g., (B) H.C.H. catalyst 2 g., and Monomer C $44 \mathrm{ml}$. Dissolve the catalyst in the monomer, warming if required to $37^{\circ} \mathrm{C}$. Add plasticizer $9 \mathrm{ml}$., filter through Whatman paper No. 1. Add (B) to (A) and stir gently to ensure thorough mixing. For use add $1 \mathrm{ml}$. accelerator $\mathbf{E}$ to the above mixture, stir gently and allow to stand a few minutes for any bubbles to disperse before use. $\ddagger$

Moulds.-Specimens can be embedded in almost any container which does not leak. Boxes can conveniently be made of glass cut to size and bound together with cellophane tape or sealed with Semmentum glue. Cardboard boxes lined with cellophane or straight sided tins may be used.

Casting of the Specimen.-

(a) Divide the resin mixture into two equal parts. Add the necessary amount of accelerator $E$ to one half, and after thorough dispersion, pour into a mould of convenient depth, according to the size required in the final block. This will set in about one hour at normal room temperature (it is not advisable to use a curing temperature higher than $40^{\circ} \mathrm{C}$.). . During this stage it is important to cover the mould so that the tacky surface is kept dust free.

"Marco polyester resin may be obtained from Scott Bader \& Co., Ltd., 109, Kingsway, London, W.C.2 and the technique described is based upon the instructions supplied by them.

+This mixture is stable for 1-2 days at room temperature and 4-5 days in the refrigerator.

¥ This final accelerated resin is stable for only 1-2 hours (approx.) at room temperature and 12 hours (approx.) in the refrigerator. 
(b) Carefully place the specimen on the tacky surface of the first layer. Avoid touching the surface more than necessary.

(c) Take the remainder of the resin mixture, add the required amount of accelerator E, mix, and pour it slowly over the specimen. Sufficient resin should be used to submerge the specimen to a depth of at least 1 in. Care should be taken not to trap any bubbles underneath the specimen.

(d) Allow this final layer of the casting to solidify in the refrigerator overnight and leave for a further 24 hours before removing the block from the mould.

(e) Wash the sticky surface with acetone. Cut the block to the size required and then rub down the surface with suitable grades of emery paper. Leave for a few days to set completely hard and polish with a fine abrasive and high-speed mop.

Pre-casting Treatment of the Specimen.-

For opaque specimens: (a) fix in 10 per cent. Formol saline;

(b) when casting, dry the surface of the specimen with absorbent material, and allow to dry in the air until it is just short of darkening;

(c) block in resin (Fig. 7).

For cleared specimens: (a) dehydrate with graded alcohols;

(b) leave in chloroform 24 hours;

(c) Clear in monomer $\mathrm{C}$;

(d) block in resin (Fig. 8).

My thanks are due to, Dr. Peter Hansell for the photographs. I am particularly indebted to my chief technician, Mr. G. E. Knight, who has largely been responsible for investigating these methods, and to Mr. D. Bedford and Mr. G. Sharman for further technical assistance.

\section{REFERENCES}

Hacketr, C. J., and Norman, W. A. (1950). Trans. R. Soc. trop. Med. Hyg., 43, 357.

\section{NOTES}

Honours THE Council of the Ophthalmological Society of the United Kingdom announces that the Edward Nettleship Prize for 1950 has been awarded to Professor A. J. Ballantyne, LL.D., M.D., F.R.F.P.S.G.

Fellowship THE Faculty of Medicine, University of Toronto, announces the renewal of the Fellowship in Ocular Genetics of the Canadian National Institute for the Blind. The grant is $\$ 2,000$ for research in this field. This Fellowship was held during 1949-1950 by Dr. L. A. Probert, of Moose Jaw, Saskatchewan, who has been carrying on studies of the hereditary aspects of glaucoma. Dr. G. A. Thompson, of Richmond Hill, Ontario, has been appointed to continue these studies in 1951 through funds provided by this Fellowship. 


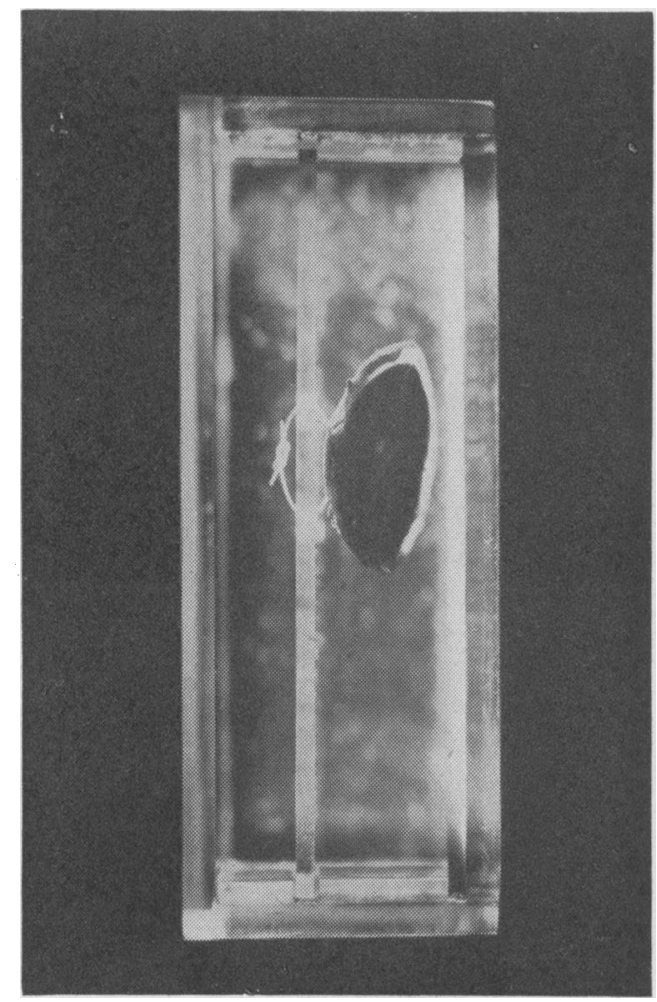

FIG. 3.-The specimen is mounted on the centre plate and tied into position with superfine Nylon sutures.

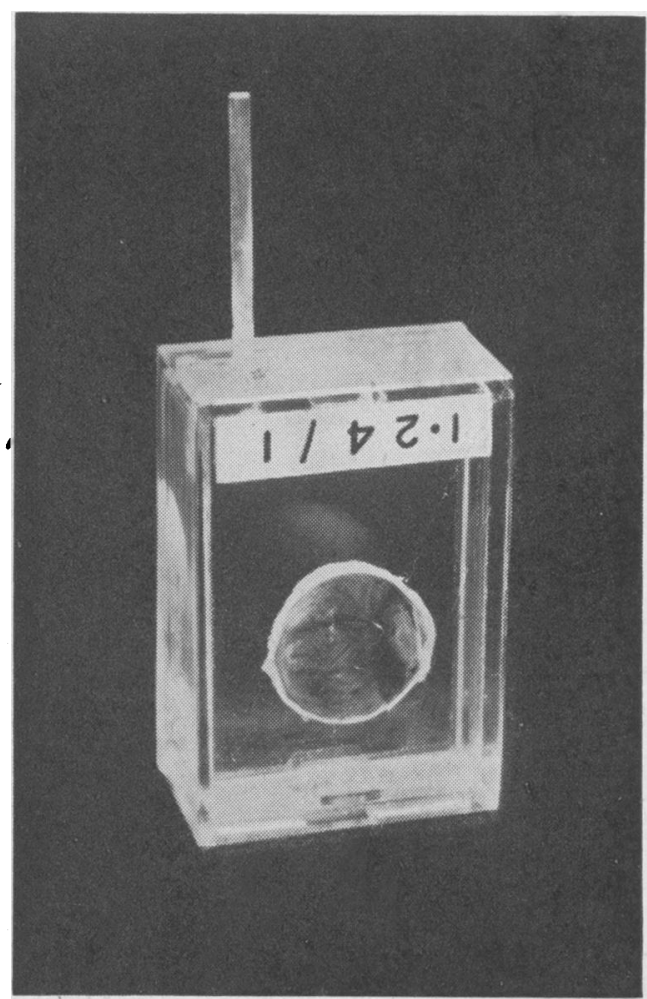

FIG. 4.-The box is filled through a hole in the bottom plate, which is later sealed with a Perspex plug. 